\title{
Volume Uterino em Adolescentes Avaliado pela Ultra-sonografia
}

\author{
Uterine Volume in Teenagers Evaluated by Ultrasound
}

Francisco Mauad Filho, Antonio Gadelha da Costa, Patricia Spara, Adilson Cunha Ferreira, Luciano Pinheiro Filho, Fernando Marum Mauad, Glauce Gelonezi

\section{RESUMO}

Objetivo: avaliar o volume uterino de mulheres entre 10 e 40 anos, verificando-se se o volume uterino de adolescentes é menor que o volume uterino de mulheres entre 20 e 40 anos. Procuram-se enfatizar as diferenças entre o volume uterino de adolescentes e mulheres adultas correlacionando-as com a imaturidade do trato genital de adolescentes para a gravidez e o parto.

Métodos: estudo transversal, no qual 828 pacientes entre 10 e 40 anos foram divididas em dois grupos e avaliadas por meio da ultra-sonografia transabdominal para aferição do volume uterino. O primeiro grupo (Ad) foi formado por 477 (57,7\%) adolescentes e o segundo grupo (Ma) por 351 (42,3\%) mulheres adultas entre 20 e 40 anos. No grupo Ad, os exames ultrasonográficos foram realizados por um único observador e no grupo $\mathrm{Ma}$, por um grupo de médicos que seguiram a mesma metodologia utilizada no grupo Ad. Os aparelhos ultrasonográficos utilizados foram Image Point HX (Hewlett Packard) e Hitachi 525, com transdutor convexo multifreqüencial. O cálculo do volume uterino foi obtido pelos diâmetros longitudinal $(D L)$, ântero-posterior (DAP) e transverso (DT), multiplicados pela constante 0,45 .

Resultados: o volume uterino de adolescentes entre 10 e 17 anos foi menor que o volume uterino de mulheres entre 20 e 40 anos $(p<0,05)$. Adolescentes secundiparas apresentaram volume uterino semelhante ao volume uterino de mulheres secundiparas entre 20 e 40 anos $(62,6 \pm 20,6$ e 69,0 $\pm 22,9)(p>0,05)$.

Conclusão: o volume uterino de adolescentes com menos de 18 anos ou primíparas é menor que o volume uterino de mulheres entre 20 e 40 anos. Entretanto, adolescentes com 18 anos ou mais, ou secundiparas, têm volume uterino similar ao volume uterino de mulheres entre 20 e 40 anos.

PALAVRAS-CHAVE: Volume uterino. Adolescência. Menarca.

\section{Introdução}

Cronologicamente, a adolescência corresponde à idade entre 10 a 19 anos $^{1}$ e é neste período que se insere todo o processo de desenvolvimento sexual, caracterizado pela telarca, pubarca e menarca, o que constitui a primeira fase da adolescência, denominada puberdade. A menina passa a experimentar importantes modifica-

Departamento de Ginecologia e Obstetricia da Faculdade de Medicina de Ribeirão Preto da Universidade de São Paulo Francisco Mauad Filho

Av. dos Bandeirantes, 3.900 - $8^{\circ}$ andar - Campus Universitário

14049-900 - Ribeirão Preto - SP

Fone: (16) 602-2587

e-mail: gadelhamail@bol.com.br ções físicas e fisiológicas, que se caracterizam pelo crescimento do mamilo e pigmentação da aréola (telarca), aparecimento dos pelos pubianos (pubarca), primeira menstruação (menarca) e, ao mesmo tempo, crescimento e desenvolvimento do tecido celular subcutâneo, dando ao corpo os típicos traços femininos. Seguem-se a este período o menacme, período em que a mulher alcança a maturidade plena e torna-se apta para o fenômeno da reprodução, e o climatério, que corresponde à transição entre o período reprodutivo e o não reprodutivo ${ }^{2}$.

As mudanças no comportamento e nos hábitos da população na vida moderna trouxeram consigo profundas modificações na adolescente, que se fizeram refletir na sua conduta sexual e conseqüentemente na sua vida reprodutiva. A li- 
beração sexual a partir dos anos 60 tornou as relações sexuais mais precoces e mais freqüentes, com conseqüente aumento no número de gestações em adolescentes, fenômeno observado em diversos países $^{3}$. Em países desenvolvidos, como por exemplo, a Inglaterra, em 1997, mais de 90.000 adolescentes engravidaram, das quais 8.000 tinham idade inferior a 16 anos e 2.200 eram inferiores a 14 anos $^{4}$. No Brasil, segundo a Pesquisa de Demografia e Saú$\mathrm{de}^{5}, 18 \%$ das adolescentes já tiveram um filho ou estão grávidas. Em Ribeirão Preto, entre 1968 e 1970, $11,7 \%$ de nascidos vivos foram de mães adolescentes $^{6}$, aumentando para $14,1 \%$ entre 1978 e $1979^{7}$.

Espera-se nas gestações que ocorrem neste período maior número de implicações físicas e sociais, pois atinge a mulher em fase de profundas transformações. Portanto, torna-se cada vez mais claro que a gravidez na adolescência é primariamente um problema social que pode acarretar importantes complicações médicas ${ }^{8}$. As grávidas adolescentes apresentam grandes riscos de complicações durante a gravidez e o parto, apresentando, portanto, maiores riscos de mortalidade materna e perinatal ${ }^{9}$. Em face disto, as complicações do parto e puerpério constituem a principal causa de morte na faixa etária de 15 a 19 anos, principalmente nos estados hipertensivos, infecções puerperais, hemorragias e abortos, que se agravam, se não houver assistência pré-natal adequada $^{10}$.

Do mesmo modo, mães adolescentes têm maior probabilidade de terem filhos com baixo peso ao nascer, como também maiores índices de partos pré-termos. Adolescentes entre 13 e 15 anos apresentam quase cinco vezes mais aumento do risco de parto pré-termo, na primeira gravidez, do que mulheres entre 20-24 anos ${ }^{11}$. Desta forma, fatores biológicos, tais como imaturidade fisiológica e desenvolvimento incompleto da pelve feminina e do útero, têm papel preponderante no aumento de complicações maternas e perinatais nas grávidas adolescentes ${ }^{12}$.

Assim sendo, o objetivo do presente estudo é determinar o volume uterino de mulheres entre 10 e 40 anos por meio da ultra-sonografia, verificando-se se a idade e a menarca têm influência sobre o volume uterino de adolescentes. Verifica-se também se o volume uterino de adolescentes é menor que o volume uterino de mulheres entre 20 e 40 anos, considerando-se a mesma paridade para os dois grupos. Tendo em vista a maior incidência de parto pré-termo em adolescentes que em mulheres adultas e considerandose a idade e a paridade nos dois grupos, procurase enfatizar as diferenças entre o volume uterino de adolescentes e mulheres adultas.

\section{Pacientes e Métodos}

Foi realizado estudo prospectivo transversal, no qual 828 pacientes, com idade entre 10 e 40 anos, foram avaliadas pela ultra-sonografia para aferição do volume uterino. As pacientes, oriundas da rede de Saúde Pública do Município de Ribeirão Preto, foram atendidas no período de maio de 2001 a maio de 2002, na Escola de UltraSonografia e Reciclagem Médica de Ribeirão Preto. Este trabalho foi aprovado pelo Comitê de Ética em Pesquisa do Hospital das Clínicas da Faculdade de Medicina de Ribeirão Preto da Universidade de São Paulo.

As pacientes foram selecionadas conforme os critérios de inclusão e exclusão. Os critérios de inclusão foram pacientes com idade entre 10 e 40 anos (nuligestas, primíparas e secundiparas), com úteros normais à ecografia pélvica e concordância acerca da realização da ultra-sonografia pélvica por via abdominal. Foram excluídas do estudo grávidas, pacientes com malformações uterinas, miomas, pólipos, adenomiose e antecedentes de cirurgia uterina prévia. Todas as pacientes assinaram o termo de consentimento informado.

A princípio, estabeleceram-se dois grupos para nossa amostra. O primeiro grupo (Ad) foi formado por $477(57,7 \%)$ adolescentes e o segundo grupo (Ma), por $351(42,3 \%)$ mulheres adultas entre 20 e 40 anos.

As hipóteses a serem testadas foram: verificar se a idade e a menarca tinham influência no volume uterino de adolescentes; testar se o volume uterino do grupo Ad é diferente do volume uterino do grupo Ma, estabelecendo-se a mesma paridade para os dois grupos, e observar se dentre o grupo de adolescentes nuliparas havia uma faixa etária em que o volume uterino era semelhante ao volume uterino de mulheres entre 20 e 40 anos.

As pacientes do grupo Ad foram avaliadas quanto à presença de menarca e quanto à paridade, sendo que para esta última variável as pacientes foram divididas em nuligestas (P0), primíparas (P1) e secundiparas (P2). As pacientes entre 20 e 40 anos, segundo a variável paridade, também foram divididas em nuligestas (P0), primíparas ( $\mathrm{P} 1)$ e secundiparas (P2).

Os exames ultra-sonográficos foram realizados por via abdominal utilizando aparelhos modelos Image Point HX (Hewlett Packard) e Hitachi 525, com transdutor convexo abdominal multifreqüencial. Antes do procedimento, as pacientes foram orientadas a tomar 3 a 4 copos de água e não urinar até 1 hora antes da realização do exame, para se obter adequada repleção vesical, que era 
considerada satisfatória para visualizar o fundo uterino, sem a presença de sombra acústica. As pacientes foram posicionadas em decúbito dorsal e após aplicação do gel sob o transdutor, procedia-se à aferição do volume uterino, estabelecendo-se como variáveis para seu cálculo o diâmetro longitudinal (DL), o diâmetro ântero-posterior (DAP) e o diâmetro transverso (DT), que foram aplicados à fórmula (DL x DAP x DT) x 0,45 ${ }^{13}$.

As variáveis analisadas foram volume uterino, idade, menarca e paridade. Os dados obtidos foram tabulados no programa Microsoft Excel e para análise estatística dos resultados foram utilizados o coeficiente de correlação de Pearson, a análise de variância, o teste $t$ de Student, o teste de Mann-Whitney e o teste post hoc de Bonferroni. Para significância estatística considerou-se $\mathrm{p}<0,05$.

\section{Resultados}

Para a primeira hipótese testada, aplicamos o coeficiente de correlação de Pearson, que foi 0,52, mostrando correlação imperfeita positiva ${ }^{14}$ entre a idade e o volume uterino na faixa etária de 10 a 40 anos, isto é, à medida que a idade aumenta o volume uterino também aumenta. Avaliando o grupo de adolescentes entre 10 e 19 anos pelo mesmo procedimento, o coeficiente de correlação de Pearson foi 0,26, demonstrando haver, também, correlação imperfeita positiva ${ }^{14}$ entre idade e volume uterino nesta faixa etária, porém menos significante que a observada no grupo de mulheres entre 10 e 40 anos. Estes resultados estão apresentados nas Figuras 1 e 2, representando modelo linear que, por meio do método de análise de regressão, quantifica esta relação.

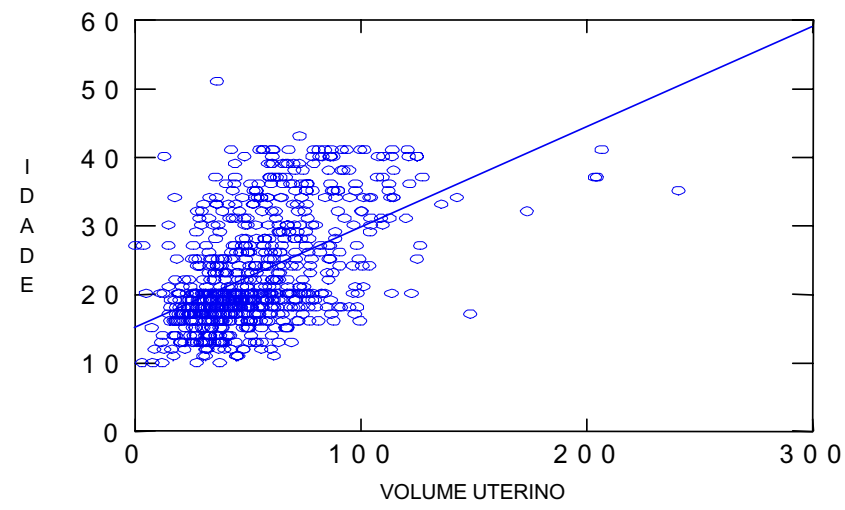

Figura 1 - Relação entre a idade e o volume uterino em 828 pacientes entre 10 e 40 anos. Dados apresentados em $\mathrm{cm}^{3}$. Coeficiente de correlação de Pearson $(r=0,52)$. Equação de regressão linear: $\mathrm{VU}=13,05+1,84 \times$ idade, $p<0,001$.

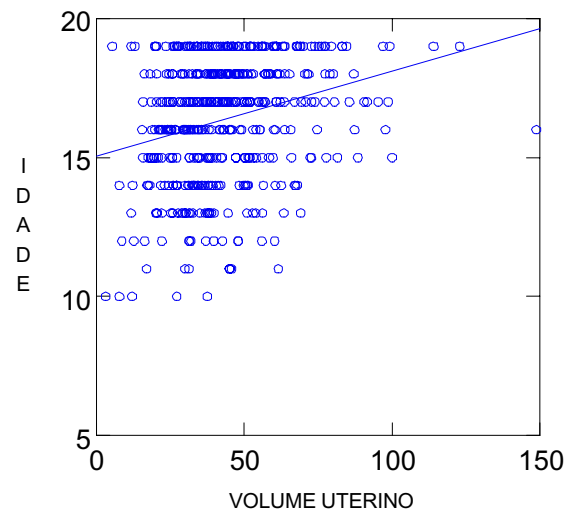

Figura 2 - Relação entre a idade e o volume uterino em 477 pacientes entre 10 e 19 anos. Dados apresentados em $\mathrm{cm}^{3}$. Coeficiente de correlação de Pearson $(r=0,26)$ Equação de regressão linear: $\mathrm{VU}=6,23+2,26 \times$ idade, $p<0,001$.

Com relação à influência da menarca sobre o volume uterino de adolescentes verificou-se que as adolescentes que já haviam tido menarca apresentavam volume uterino superior às que não tinham tido menarca (os valores foram de 40,73 $\pm 12,91$ e 22,1 $\pm 18,32$, respectivamente); teste MannWhitney $-\mathrm{p}=0,04)$. Como foi demonstrado que a idade tinha influência sobre o volume uterino, este resultado foi obtido apenas no grupo com menos de 13 anos, tendo em vista este ser o único grupo de adolescentes que não mostrou a interferência da idade sobre o volume uterino (teste de MannWhitney $-\mathrm{p}=0,11$ ).

Para testarmos a segunda hipótese, a princípio, avaliamos o volume uterino das pacientes entre 10 e 40 anos de acordo com a paridade, classificando-as em PO (nuligestas), P1 (primíparas) e P2 (secundiparas). Utilizando a técnica de análise de variância e o teste post hoc de Bonferroni, verificamos que o volume uterino aumenta com a paridade, de modo que $\mathrm{P} 0<\mathrm{P} 1<\mathrm{P} 2$. Em seguida, procuramos estabelecer se as adolescentes entre 10 e 19 anos apresentavam volume uterino menor do que as mulheres entre 20 e 40 anos, considerando a mesma paridade para os dois grupos. Observamos que para os subgrupos $\mathrm{PO}$ e $\mathrm{P} 1$ o volume uterino de adolescentes foi significantemente menor que em mulheres entre 20 e 40 anos ( $<<0,001)$. Adolescentes nuligestas apresentaram volume uterino de $41,3 \pm 17,9 \mathrm{~cm}^{3}$, ao passo que adolescentes primiparas tinham volume uterino de $51,6 \pm 19,7$ $\mathrm{cm}^{3}$ (Tabela 1). No subgrupo P2, o volume uterino das adolescentes entre 10 e 19 anos não mostrou diferença significante em relação ao volume uterino das pacientes entre 20 e 40 anos $(p=0,22)$, tendo sido $62,6 \pm 20,6 \mathrm{~cm}^{3}$ nas adolescentes e $69,0 \pm 22,9 \mathrm{~cm}^{3}$ nas mulheres entre 20 e 40 anos (Tabela 1). É evidente que, como já descrito, as variáveis idade e menarca podem ter influenciado no nível de significância observado nesta última aná- 
lise. Para verificarmos esta proposição, utilizamos análise de regressão multivariada para as variáveis idade, paridade e menarca, obtendo-se significância estatística $(p<0,05)$. A equação da análise de regressão multivariada ficou assim representada: volume uterino $=8,887+1,138 \mathrm{x}$ idade $+5,894$ $\mathrm{x}$ paridade $+17,134 \mathrm{x}$ menarca. Verificou-se, portanto, que o maior volume uterino foi encontrado na maior idade e maior paridade e que o menor volume uterino foi encontrado na menor idade e menor paridade (Tabela 1).

Tabela 1 - Comparação entre o volume uterino de adolescentes entre 10 e 19 anos com mulheres entre 20 e 40 anos, considerando, em cada análise, a mesma paridade para os dois grupos.

\begin{tabular}{lc}
\hline Pacientes & Volume uterino $\left(\mathrm{cm}^{3}\right)$ \\
\hline $10-19-P 0(n=407)$ & $41,3 \pm 17,9$ \\
$20-40-P 0(n=109)^{*}$ & $52,1 \pm 22,0$ \\
$0-19-P 1(n=47){ }^{* *}$ & $51,6 \pm 19,7$ \\
$20-40-P 1(n=65)$ & $64,5 \pm 24,3$ \\
$10-19-P 2(n=21)$ & $62,6 \pm 20,6$ \\
$20-40-P 2(n=77)$ & $6 * * 0 \pm 22,9$ \\
\hline
\end{tabular}

Medidas em média \pm desvio padrão.

P0: nulíparas; P1: primíparas; P2: secundíparas.

${ }^{*} p<0.001$ teste $t$ de Student - comparou nulíparas 10-19 anos com nulíparas 20-40 anos.

${ }^{* *} p<0.003$ teste $t$ de Student - comparou primíparas 10-19 anos com primiparas 20-40 anos.

${ }^{* * *} p=0.22$ teste $t$ de Student - comparou secundíparas 10-19 anos com secundíparas 20-40 anos.

Finalmente, procuramos verificar entre as adolescentes nuligestas se havia uma faixa etária na qual o volume uterino fosse semelhante ao de mulheres nuligestas entre 20 e 40 anos. Para esta proposição as pacientes foram divididas em 6 subgrupos de acordo com a faixa etária (em anos): 10 e 11 (subgrupo 1), 12 e 13 (subgrupo 2), 14 e 15 (subgrupo 3), 16 e 17 (subgrupo 4), 18 e 19 (subgrupo 5) e 20 a 40 (subgrupo 6). Por meio do teste post hoc de Bonferroni, observou-se que o volume uterino das adolescentes entre 10 e 17 anos foi menor que o volume uterino das mulheres entre 20 e 40 anos $(\mathrm{p}=0,05)$ (Tabela 2). Por outro lado, o volume uterino das adolescentes entre 18 e 19 anos foi semelhante ao das pacientes entre 20 e 40 anos, cujos valores foram, respectivamente, $44,8 \pm 17,8 \mathrm{~cm}^{3} \mathrm{e}$ $50,3 \pm 21,0 \mathrm{~cm}^{3}(\mathrm{p} \geq 0,05)$ (Tabela 2).

\section{Discussão}

A evolução tecnológica tornou possivel o desenvolvimento de aparelhos ultra-sonográficos sofisticados, que permitem a aferição precisa do volume uterino, de modo que este método tem sido amplamente utilizado para o conhecimento morfofuncional do útero. Nesta linha de pesquisa, podemos citar o estudo de Gross et al. ${ }^{13}$, no qual relataram que a medida do volume do útero, avaliada pela ultra-sonografia bidimensional, era semelhante à encontrada quando aferida por um paquímetro de precisão em diversas faixas etárias, demonstrando a confiabilidade deste método na determinação do volume uterino. Em relação à ultra-sonografia pélvica para a aferição do volume uterino, está estabelecido na literatura que não há diferença significante quando o mesmo é obtido por via abdominal ou endovaginal ${ }^{15,16}$.

Tabela 2 - Comparação entre o volume uterino de nulíparas adolescentes entre 10-17 anos e 18-19 anos com nulíparas entre 20 e 40 anos.

\begin{tabular}{lc}
\hline Pacientes & Volume uterino $\left(\mathbf{c m}^{3}\right)$ \\
\hline $10-11-S 1(n=12)$ & $30,2 \pm 17,8$ \\
$12-13-S 2(n=45)$ & $35,7 \pm 13,9$ \\
$14-15-S 3(n=91)$ & $39,6 \pm 16,7$ \\
$16-17-S 4(n=120)$ & $40,0 \pm 16,5$ \\
$20-40-G 2(n=92) *$ & $50,3 \pm 21,0$ \\
$18-19-S 5(n=119)$ & $44,8 \pm 17,8$ \\
$20-40-G 2(n=92)^{* *}$ & $50,3 \pm 21,0$
\end{tabular}

Medidas em média \pm desvio padrão.

S: subgrupo; G: grupo.

${ }^{*} p<0,05$ - análise de variância e teste post hoc de Bonferroni - comparou nulíparas adolescentes entre 10 e 17 anos com nulíparas entre 20 e 40 anos.

${ }^{* *} p>0,05$ - análise de variância e teste post hoc de Bonferroni - comparou nulíparas adolescentes entre 18 e 19 anos com nulíparas entre 20 e 40 anos.

Entre 4 e 6 semanas de idade, o útero apresenta forma cilindrica e o volume do colo predomina sobre o do corpo. Evolui da forma infantil para a forma adulta, paulatinamente, de modo que, na menarca, começa a haver uma diminuição relativa do comprimento do colo em favor do corpo, devido ao crescimento do corpo uterino, que assume forma eliptica, dando ao útero aspecto piriforme. Fatores relacionados ao desenvolvimento normal do ciclo genital feminino podem levar a alterações no tamanho uterino ${ }^{2}$. Dentre estes, o desenvolvimento endócrino do sistema reprodutivo feminino parece ser fator importante que influencia no tamanho do útero, órgão propício de apresentar mudanças sob estimulos hormonais, de forma que este parâmetro é extremamente importante nas avaliações deste órgão. Observa-se aumento progressivo no tamanho uterino à medida que a menina passa da infância para a adolescência, tornando-se significante durante a puberdade ${ }^{17}$.

O presente trabalho demonstrou que o volume uterino aumenta proporcionalmente ao aumento da idade. Entretanto, esta relação foi mais 
significante na idade entre 20 e 40 anos que em adolescentes. $\mathrm{O}$ volume uterino nas adolescentes foi de $43,3 \pm 18,90 \mathrm{~cm}^{3}$ e na faixa etária entre $20 \mathrm{e}$ 40 anos, $66,3 \pm 30,78 \mathrm{~cm}^{3}$. São concordantes com nossos achados os relatos de Mauad Filho et al. ${ }^{16}$, de 2001, quando relataram que o volume uterino em pacientes entre 10 e 18 anos era de $39,0 \mathrm{~cm}^{3}$ e em pacientes entre 19 e 48 anos, $66,8 \mathrm{~cm}^{3}$. Dados da literatura mostram que o útero mede 26,4 $\mathrm{mm}$ em meninas com menos de 2 anos, 29,5 mm entre 2 a 8 anos, 36, $5 \mathrm{~mm}$ entre 8 e 12 anos, atingindo $60 \mathrm{~mm}$ na puberdade. Estes dados reforçam a importância da avaliação do tamanho uterino em doenças que acometem pacientes jovens como a puberdade precoce, puberdade tardia, hipopituitarismo e hiperandrogenismo ${ }^{18}$.

Parece haver forte relação entre o desenvolvimento da maturidade feminina e o volume uterino. Este estudo mostrou que o volume uterino das adolescentes que não tinham menarca era menor $\left(22,10 \pm 18,32 \mathrm{~cm}^{3}\right)$ que o volume uterino das adolescentes com menarca $\left(40,73 \pm 12,91 \mathrm{~cm}^{3}\right)$. Corroborando com nossos achados, Herter et al. ${ }^{19}$, em 2002, mostraram a relação existente entre o volume uterino e a telarca. Correlacionando o volume uterino de meninas entre 1 e 13 anos com a telarca, estes autores observaram que o mesmo foi menor em pacientes sem telarca, que nas com telarca. Nestas, o volume uterino foi de 8,1 $\pm 6,6$ $\mathrm{cm}^{3}$ e naquelas, $1,8 \pm 1,2 \mathrm{~cm}^{3}$.

Enfatizando o aspecto biológico como fator relacionado ao prognóstico de grávidas adolescentes, há relatos que a imaturidade fisiológica e o desenvolvimento incompleto do útero estão relacionados às taxas de prematuridade e mortalidade perinatal nesta faixa etária ${ }^{12}$. Em 2001, Olausson et al. ${ }^{11}$ observaram que a prematuridade extrema na primeira gravidez foi substancialmente mais alta entre adolescentes do que em mulheres entre 20 e 24 anos. Assim sendo, a mortalidade após o nascimento ocorre três vezes mais em recém-nascidos filhos de adolescentes com 15 anos ou menos, que em mulheres entre 23 e 29 anos $^{20}$. Ainda sobre o aspecto biológico, Ibanez et al. ${ }^{21}$ observaram que adolescentes nascidas de mães que tiveram restrição de crescimento intrauterino apresentavam útero menor que aquelas nascidas de mães cuja gravidez foi normal.

No presente estudo, quando se comparou o volume uterino de nuligestas entre as diversas faixas etárias do grupo Ad com o volume uterino de nuligestas do grupo Ma, verificou-se que as adolescentes com idade igual ou superior a 18 anos apresentaram volume uterino semelhante ao grupo de mulheres entre 20 e 40 anos. O volume uterino das adolescentes foi menor nas nuligestas $(\mathrm{p}<0,05)$ e primíparas $(\mathrm{p}<0,05)$. Porém, a partir de dois partos, o volume uterino na adolescência foi semelhante ao volume uterino de mulheres entre 20 e 40 anos, considerando-se a mesma paridade para os dois grupos $(p>0,05)$. Tomando por base os resultados obtidos nos fatores idade e paridade, entendemos que a semelhança encontrada no volume uterino entre os dois grupos a partir dos 18 anos e/ou quando as adolescentes são secundíparas pode estar relacionada à maturidade uterina. A maior incidência de parto pré-termo na adolescência que em mulheres adul$\operatorname{tas}^{11}$ pode estar relacionada ao volume uterino, tendo em vista que adolescentes nuligestas com idade igual ou inferior a 17 anos apresentaram volume uterino menor que mulheres nuligestas entre 20 e 40 anos.

Neste contexto, acreditamos que o presente estudo contribuirá com os programas educacionais relacionados à escolha da idade ideal da mulher para o início da vida reprodutiva, com conseqüente redução na mortalidade materna e neonatal entre grávidas adolescentes e conseqüentemente melhoria nos indicadores de saúde da população.

\section{ABSTRACT}

Purpose: to evaluate the uterine volume in women between 10 and 40 years in order to observe if the uterine volume in adolescents is smaller than the uterine volume in women between 20 and 40 years. We intend to emphasize the differences between the uterine volume of adolescents and that of adult women and to correlate with the immaturity of the genital tract of adolescents regarding gestation and delivery.

Method: a cross-sectional study, which included 828 patients between 10 and 40 years old divided into two groups and examined using abdominal ultrasound to obtain the uterine volume measure. The first group consisted of 477 (57.7\%) adolescents, and the second group of 351 (42.3\%) adult women between 20 and 40 years old. In the adolescent group, ultrasound examination was performed by a single observer and in the group of adult women ultrasound examination was performed by a group of observers who used the same methodology as that of group 1. Image Point HX (Hewlett Packard) and Hitachi 525 ultrasound equipment were used with a multiple frequency probe. For the calculation of the uterine volume we used the longitudinal diameter $(L D)$, anteroposterior diameter (APD) and transverse diameter (TD) with the (LD $\times A P D \times T D) \times 0.45$ formula.

Results: adolescents aged 10 to 17 years had a smaller uterine volume than women aged 20 to 40 years $(p<0.05)$. Adolescents who delivered twice had a uterine volume similar to that of the patients between 20 and 40 years old with respective mean values of $62.6 \pm 20.6$ and $69.0 \pm 22.9(p>0.05)$. 
Conclusion: adolescents less than 18 years old or primiparous have a smaller uterine volume than women between 20 to 40 years old. However, adolescents aged 18 years or older, or secundipara, have a uterine volume similar to that of women aged 20 to 40 years.

KEYWORDS: Uterine volume. Adolescence. Menarche.

\section{Referências}

1. Organización Panamericana de la Salud. La salud de los adolescentes y los jóvenes en las Américas: escrebiendo el futuro. Washington (DC); 1995. (Comunicación para la salud, 6).

2. Cunha DC, Salgado Neto J, Halbe HW. Fases biológicas da mulher. In: Halbe HW, editor. Tratado de Ginecologia. $3^{a}$ ed. São Paulo: Roca; 2000. p.339-42.

3. Gama SGN, Szwarcwald CL, Leal MC. Experiência de gravidez na adolescência, fatores associados e resultados perinatais entre puérperas de baixa renda. Cad Saúde Pública 2002; 18:153-61.

4. Jolly MC, Sebire N, Harris J, Robinson S, Regan L. Obstetric risks of pregnancy in women less than 18 years old. Obstet Gynecol 2000; 96:962-6.

5. CNPD. Pesquisa Nacional sobre Demografia e Saúde. Redesaude [serial online] 1997 [cited 2002 Dec 10]. Avaliable from: URL: http://www.redesaude.org.br/ dossies/html/body_ad-gravidez.html

6. Teruel JR, Gomes UA, Nogueira JL. Investigación interamericana de mortalidad en la niñez: peso al nacer en la región de Ribeirão Preto, São Paulo, Brasil. Bol Oficina Sanit Panam 1975; 79:139-45.

7. Bettiol H, Barbieri MA, Gomes UA, et al. Atenção médica à gestação e ao parto de mães adolescentes. Cad Saúde Pública 1992; 8:404-13.

8. Hollingsworth DR, Kreutner AKK. Sounding board. Teenage pregnancy: solutions are evolving. N Engl J Med 1980; 303:516-8.

9. Fraser AM, Brockert JE, Ward RH. Association of young maternal age with adverse reproductive outcomes. N Engl J Med 1995; 332:1113-7.

10.Scholl TO, Hediger ML, Belsky DH. Prenatal care and maternal health during adolescent pregnancy: a review and meta-analysis. J Adolesc Health 1994; 15:444-56.

11. Olausson PO, Cnattingius S, Haglund B. Does the increase risk of preterm delivery in teenagers persist in pregnancies after the teenage period? BJOG 2001; 108:721-5.

12.Roth J, Hendrickson J, Schilling M, Stowell DW. The risk of teen mothers having low birth weight babies: implications of recent medical research for school health personnel. J Sch Health 1998; 68:271-5.

13.Gross R, Mauad Filho F, Ayres CE, Baracchini JAA, Mangieri Sobrinho F, Ferreira AC. O volume uterino de acordo com o desenvolvimento ciclo genital da mulher. Rev Bras Ginecol Obstet 1996; 18:581-6.

14. Martins GA. Estatística geral e aplicada. $1^{\mathrm{a}}$ ed. São Paulo: Atlas; 2001. p.288.

15. Nazario ACP, Nicolau SM, Nishimura CM. Comparison between pelvic endovaginal and transabdominal sonography in the measurement of the uterus and ovaries. Rev Paul Med 1991; 109:51-4.

16. Mauad Filho F, Beduschi AF, Meschino RAG, Mauad FM, Casanova MS, Ferreira AC. Avaliação ultrasonográfica das variações do volume uterino. Rev Bras Ginecol Obstet 2001; 23:175-9.

17.Buzi F, Pilotta A, Dordoni D, Lombardi A, Zaglio S, Adlard P. Pelvic ultrasonography in normal girls and in girls with pubertal precocity. Acta Paediatr 1998; 87:1138-45.

18. Colle M, Calabet A, Cadier L, Battin J. Pelvic echographic data during normal and pathological development in girls. Arch Fr Pediatr 1984; 41:405-8.

19.Herter LD, Golendziner E, Flores JA, Becker E Jr, Spritzer PM. Ovarian and uterine sonography in healthy girls between 1 and 13 years old: correlation of findings with age and pubertal status. AJR Am J Roentgenol 2002; 178:1531-6.

20.Phipps MG, Blume JD, DeMonner SM. Young maternal age associated with increased risk of postneonatal death. Obstet Gynecol 2002; 100:481-6.

21.Ibanez L, Potau N, Enriquez G, de Zegher F. Reduced uterine and ovarian size in adolescent girls born small for gestational age. Pediatr Res 2000; 47:575-7. 